\title{
AN INEQUALITY FOR ANALYTIC FUNCTIONS
}

\section{HERBERT KAMOWITZ}

ABSTRACT. If $F$ denotes the boundary value of a function $f \in H^{p}$, $1 \leq p \leq \infty$, the infimum of the measure of $\{\theta|| F(\theta) \mid>A\}$ for given $A$, $0<A<|f(0)|,\|f\|_{H^{p}}=1$, is determined.

In this note we discuss an aspect of the boundary behaviour of certain functions analytic on the unit disc in terms of their values at the origin. Specifically, if $F(\theta)$ is the boundary value of a function $f \in H^{p}, 1 \leq p \leq \infty$, and if a number $A, 0<A<|f(0)|$, is chosen, we are interested in minimizing the measure of $\{\theta \mid 0 \leq \theta \leq 2 \pi$ and $|F(\theta)|>A\}$ over all $f \in H^{p}$ with $\|f\|_{H^{p}}=1$. The result is that, for $p<\infty$, the infimum is the solution $c$ of the equation

$$
c \log \left[1+2 \pi\left(1-A^{p}\right) / c A^{p}\right]=2 \pi p \log (|f(0)| / A),
$$

while if $p=\infty$, the infimum is $2 \pi(1-\log |f(0)| / \log A)$, which is the limit, as $p \rightarrow \infty$, of the solutions of (1).

The ingredients of the proof are Jensen's inequality and Jensen's formula which we state in the following forms.

1. Jensen's inequality. If $\mu$ is a positive measure on a measure space $X$ with $\mu(X)=1$, and if $f \in L^{1}(d \mu)$, then

$$
\int_{X} \log |f| d \mu \leq \log \int_{X}|f| d \mu .
$$

Equality holds if, and only if, $f$ is an outer function [1, p. 62].

2. Jensen's formula. If $f \in H^{1}$ of the unit disc and $F(\theta)=\lim _{r \uparrow 1} f\left(r e^{i \theta}\right)$, then

$$
\log |f(0)| \leq \frac{1}{2 \pi} \int_{0}^{2 \pi} \log |F(\theta)| d \theta
$$

Equality holds if, and only if, $f$ is an outer function [1, p. 62].

We begin by showing, in Lemma 1 , that for each pair of numbers $A_{0}, A$, $0<A<A_{0}<1$, there is a step function $h$ on $(0,2 \pi)$ satisfying $\int_{0}^{2 \pi}|b|=2 \pi$ and $\int_{0}^{2 \pi} \log |h|=2 \pi \log A_{0}$, and which takes on the value $A$ and one other

Presented to the Society, January 18, 1974; received by the editors July 30 , 1973.

AMS (MOS) subject classifications (1970). Primary 30A40, 30A78. 
value $\bar{y}$. For $H^{1}$, the number $c=$ measure $\{x \mid h(x)=\bar{y}\}$ will be the required solution of (1).

Lemma 1. Given $0<A<A_{0}<1$, there exist unique numbers $c$ and $\bar{y}$ (depending on $A$ and $A_{0}$ ) such that $0<c<2 \pi, 1<\bar{y}$ and

(2) $2 \pi=(2 \pi-c) A+c \bar{y}$ and $2 \pi \log A_{0}=(2 \pi-c) \log A+c \log \bar{y}$.

Further, $c$ satisfies the equation

$$
c \log [1+2 \pi(1-A) / c A]=2 \pi \log \left(A / A_{0}\right) .
$$

Also, for fixed $A$, the left-hand side of (3) is an increasing function of c.

Proof. If $\bar{y}$ is the unique solution of

$$
\frac{y-A}{1-A}=\frac{\log y-\log A}{\log A_{0}-\log A}
$$

for $y>A$, then $\bar{y}>1$. Let

$$
c=2 \pi(1-A) /(\bar{y}-A) .
$$

Since equations ( $\left.4^{\prime}\right)$ with $y=\bar{y}$ and $\left(4^{\prime \prime}\right)$, together, are equivalent to equations (2), the pair $c, \bar{y}$ satisfies (2). Then from ( $4^{\prime \prime}$ ), $0<c<2 \pi$.

Equation (3) follows easily from (2) and a routine calculus argument proves the last statement of the lemma.

Lemma 2. If $0<c<2 \pi, G \in L^{1}(0,2 \pi)$ and $|G| \leq A<1$ on a set $S$ of measure $2 \pi-c$, then

$$
(2 \pi-c) A-\int_{S}|G| \leq(2 \pi-c) \log A-\int_{S} \log |G| .
$$

Proof. This follows immediately from the inequality $\log A-A \geq \log t-$ $t$ for $0<t \leq A<1$.

Lemma 3. Suppose $0<A<A_{0}<1$ and $c, \bar{y}$ are the constants satisfying equations (2) of Lemma 1. Let $F \in L^{1}(0,2 \pi)$ with $(1 / 2 \pi) \int_{0}^{2 \pi}|F|=1$ and $(1 / 2 \pi) \int_{0}^{2 \pi} \log |F|=\log A_{0}$. If $|F| \leq A$ on a set $S$ of measure $(2 \pi-c)$, then $|F|=A$ on $S$ and $|F|=\bar{y}$ off $S$.

Proof. Let $S^{\prime}=[0,2 \pi]-S$. Then

$$
\int_{S}|F|+\int_{S^{\prime}}|F|=2 \pi \text { and } \int_{S} \log |F|+\int_{S^{\prime}} \log |F|=2 \pi \log A_{0^{\circ}}
$$

From equations (2), $c$ and $\bar{y}$ satisfy

$$
2 \pi=(2 \pi-c) A+c \bar{y} \text { and } 2 \pi \log A_{0}=(2 \pi-c) \log A+c \log \bar{y} .
$$

Hence 


$$
\int_{S},|F|=(2 \pi-c) A+c \bar{y}-\int_{S}|F|
$$

and

$$
\int_{S^{\prime}} \log |F|=(2 \pi-c) \log A+c \log \bar{y}-\int_{S} \log |F| \text {. }
$$

Let

(5) $\quad a=(2 \pi-c) A-\int_{S}|F|$ and $. b=(2 \pi-c) \log A-\int_{S} \log |F|$.

Then

$$
\int_{S^{\prime}}|F|=a+c \bar{y} \text { and } \int_{S^{\prime}} \log |F|=b+c \log \bar{y}
$$

and also from Lemma $2,0 \leq a \leq b$. Now, by Jensen's inequality

$$
\int_{S}, \log |F| \frac{d \theta}{c} \leq \log \int_{S^{\prime}}|F| \frac{d \theta}{c},
$$

so that $a / c+\log \bar{y} \leq b / c+\log \bar{y} \leq \log (a / c+\bar{y})$. Since $\bar{y}>1$, it follows that $a=b=0$.

Therefore,

$$
\int_{S^{\prime}}|F| \frac{d \theta}{c}=\bar{y} \text { and } \int_{S^{\prime}} \log |F| \frac{d \theta}{c}=\log \bar{y},
$$

and the uniqueness part of Jensen's inequality implies $|F|=\bar{y}$ on $S^{\prime}$.

Also, since $a=b=0$, equations (5) imply

$$
\int_{S}|F|=(2 \pi-c) A \text { and } \int_{S} \log |F|=(2 \pi-c) \log A,
$$

and so for the same reason, $|F|=A$ on $S$.

Notation. If $S$ is a subset of $[0,2 \pi], m(S)$ will denote the Lebesgue measure of $S$.

Theorem A. Let $f \in H^{1}$ and $\|f\|_{H^{1}}=1$. Suppose $0<A<|f(0)|$ and let $c$ be the solution of

$$
c \log [1+2 \pi(1-A) / c A]=2 \pi \log (|f(0)| / A) .
$$

If $F(\theta)=\lim _{r \uparrow 1} f\left(r e^{i \theta}\right)$, then $m(\{\theta|| F(\theta) \mid>A\})$ is greater than $c$.

Proof. If $f$ is an outer function, then $(1 / 2 \pi) \int_{0}^{2 \pi}|F|=1$ and by Jensen's formula,

$$
\frac{1}{2 \pi} \int_{0}^{2 \pi} \log |F|=\log |f(0)| \text {. }
$$

Therefore, by Lemma 3, $m(\{\theta|| F(\theta) \mid>A\})$ is bigger than $c$.

If $f$ is not an outer function, write $f=B S g$, where $B$ is the Blaschke product of the zeros of $f, S$ is the singular part of $f$, and $g$ is an outer function [1]. If $G(\theta)=\lim _{r \uparrow 1}\left|g\left(r e^{i \theta}\right)\right|$, then $|G(\theta)|=|F(\theta)|$ a.e., so that 
$m(\{\theta|| G(\theta) \mid>A\})=m(\{\theta|| F(\theta) \mid>A\})$. Also since $g$ is an outer function we have $|g(0)|>|f(0)|$. By Lemma 1 , we have that the solution $c$ of $c \log [1+2 \pi(1-A) / c A]=2 \pi \log (|g(0)| / A)$ is bigger than the solution $c$ of (6) concluding the proof.

We remark that the number $c$ satisfying (6) is the best possible, since we can find $g \in H^{1}$ such that $|G|$ is arbitrarily close to the step function $H: H(\theta)=A, 0 \leq \theta<2 \pi-c, H(\theta)=\bar{y}, 2 \pi-c \leq \theta<2 \pi$.

Now, if $f \in H^{p}, 1 \leq p<\infty$, then $f^{p} \in H^{1}$ and $m(\{\theta|| F(\theta) \mid>A\})=$ $m\left(\left\{\left.\theta|| F(\theta)\right|^{p}>A^{p}\right\}\right)$. This reduces to the $H^{1}$ case with $f$ replaced by $f^{p}$ and $A$ by $A^{p}$. Hence,

Theorem B. Let $f \in H^{p}, 1 \leq p<\infty$, and $\|f\|_{H^{p}}=1$. If $0<A<|f(0)|$, then $m(\{\theta|| F(\theta) \mid>A\})$ is greater than the solution $c$ of

$$
c \log \left[1+2 \pi\left(1-A^{p}\right) / c A^{p}\right]=2 \pi p \log (|f(0)| / A)
$$

and this is the best possible.

Finally, for $H^{\infty}$ (or the disc algebra), if $f \in H^{\infty}$ and $\|f\|_{\infty} \leq 1$, we have

$$
2 \pi \log |f(0)| \leq \int_{|F| \leq A} \log |F|+\int_{|F|>A} \log |F| \leq \int_{|F| \leq A} \log |F|
$$

since $\log |F|<0$.

Therefore, $2 \pi \log |f(0)| \leq \log A \cdot m(\{\theta|| F(\theta) \mid \leq A\})$, or

$$
2 \pi \frac{\log |f(0)|}{\log A} \geq m(\{\theta|| F(\theta) \mid \leq A\})=2 \pi-m(\{\theta|| F(\theta) \mid>A\}),
$$

or

$$
m(\{\theta|| F(\theta) \mid>A\}) \geq 2 \pi(1-\log |f(0)| / \log A)
$$

Again it is easy to see that this is the best possible.

Remarks. (I) The same methods apply, as well, to any positive measure $\mu$ on a measure space $X$ for which Jensen's formula $\log \left|\int_{X} f d \mu\right| \leq \int_{X} \log |f| d \mu$ holds. (See [1, pp. 54-57], for some examples.) For the se cases, equations (1) and (7) are valid if $2 \pi$ is replaced by $\mu(X)$.

(II) By considering the Poisson kernel, it follows from (I) that we can solve the same problem in the unit.disc knowing $f$ at some point $z^{\prime}$ other than 0. A particular result is that, if $f \in H^{\infty}$ of the unit disc, $F$ is the boundary value of $f,\left|z^{\prime}\right|<1$ and $0<A<\left|f\left(z^{\prime}\right)\right|$, then the Lebesgue measure of $\{\theta|| F(\theta) \mid \leq A\}$ is less than or equal to 


$$
4 \tan ^{-1}\left[\frac{1+\left|z^{\prime}\right|}{1-\left|z^{\prime}\right|} \tan \frac{\pi}{2} \frac{\log \left|f\left(z^{\prime}\right)\right|}{\log A}\right] \text {. }
$$

I should like to thank the Pure Mathematics Department at the Weizmann Institute of Science, Rehov.ot, Israel, for the ir hospitality while this paper was being prepared.

\section{REFERENCE}

1. K. Hoffman, Banach spaces of analytic functions, Prentice-Hall Series in Modern Analysis, Prentice-Hall, Englewood Cliffs, N. J., 1962. MR 24 \#A2844.

DEPARTMENT OF MATHEMATICS, UNIVERSITY OF MASSACHUSETTS, BOSTON, MASSACHUSETTS 02125 